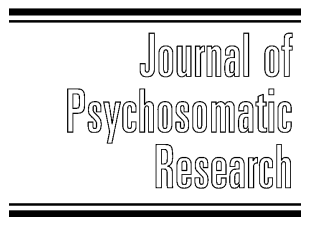

\title{
Psychosocial consequences of developmental prosopagnosia: A problem of recognition
}

\author{
Lucy Yardley $^{\mathrm{a}, *}$, Lisa McDermott ${ }^{\mathrm{a}}$, Stephanie Pisarski $^{\mathrm{a}}$, Brad Duchaine $^{\mathrm{b}}$, Ken Nakayama $^{\mathrm{c}}$

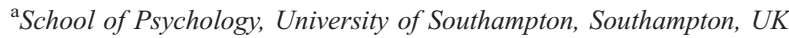 \\ ${ }^{\mathrm{b}}$ Department of Psychology, University College London, London, UK \\ ${ }^{\mathrm{c}}$ Department of Psychology, Harvard University, Boston, MA, USA
}

Received 13 December 2007; received in revised form 11 March 2008; accepted 20 March 2008

\begin{abstract}
Objective: To provide the first systematic in-depth description of the consequences of developmental prosopagnosia (DP; 'face blindness') for psychosocial functioning and occupational disability, in order to determine what kind of professional intervention may be needed. Methods: Semi-structured telephone interviews were carried out with 25 people whose selfreports of face recognition problems were confirmed by impaired scores on the Cambridge Face Recognition Test. Thematic analysis was used to inductively identify and understand common psychosocial consequences of DP. Results: All participants described recurrent and sometimes traumatic social interaction difficulties caused by recognition problems, such as failing to recognize close friends, work colleagues, and family members. These problems often led to chronic anxiety about offending others and feelings of embarrassment, guilt, and failure. Most participants described some degree of fear and

avoidance of social situations in which face recognition was important, including family and social gatherings, and meetings at work. Long-term consequences could include dependence on others, a restricted social circle, more limited employment opportunities, and loss of self-confidence. Conclusion: The potential for negative psychosocial consequences and occupational disability posed by DP is as great as that posed by conditions which are currently afforded professional recognition and support, such as stuttering and dyslexia. Wider recognition of the problems prosopagnosia can cause could reduce anxiety about social interaction difficulties by making it easier to explain and justify recognition problems to other people, including employers. Greater professional awareness could facilitate detection and referral of those requiring support with coping with social interactions.

(C) 2008 Elsevier Inc. All rights reserved.
\end{abstract}

Keywords: Developmental prosopagnosia; Qualitative research; Quality of life; Disability; Social phobia

\section{Introduction}

Developmental prosopagnosia (DP) is a condition defined by severe difficulty in recognising familiar faces $[1,2]$. Prosopagnosia was first studied as an acquired impairment arising as a consequence of brain injury, but it is now clear that severe face recognition problems can be present from childhood in the absence of any history of serious injury or

\footnotetext{
* Corresponding author. School of Psychology, University of Southampton, Highfield, Southampton, SO17 1BG, UK. Tel.: +44 2380 594581; fax: +44 2380594597.

E-mail address: 1.yardley@soton.ac.uk (L. Yardley).
}

disease. The reasons for failing to develop normal adult face recognition skills are not currently known, but appear to often include a genetic element $[3,4]$. DP may also result from prenatal or early minor brain damage, or inadequate visual input during key developmental periods (for example, due to severe myopia, or suppression of input from the left eye in amblyopia) [5].

It is now believed that the prevalence of DP may be as high as $2 \%$ of the general population [4]. However, the condition is seldom diagnosed since people with prosopagnosia can identify people in many situations by using general appearance and manner (including hair and clothes), semantic features (e.g., bushy eyebrows), voice, and contextual cues. 
Those with lifelong face recognition problems are often unaware that face recognition is typically effortless and reliable for others. Even if individuals notice their relative deficiency in face recognition, they are unlikely to suspect that this is due to a neurological problem, since there is little public awareness of DP.

The case histories and self-reports of some people with prosopagnosia (both developmental and acquired) suggest that it can have a severe impact on people's lives, resulting in avoidance of social interaction, problems with interpersonal relations, damage to career, and even depression [1,6-9]. Since DP interferes with social interaction it might predispose some people to develop social anxiety disorder, which is characterized by fear and avoidance of social situations which have the potential to cause embarrassment or humiliation $[10,11]$. It has even been suggested that DP could contribute to some cases of social developmental disorder $[12,13]$.

There have been no previous systematic investigations into the experiences of people with DP, and so the purpose of this study was to provide the first in-depth description of the psychosocial consequences of DP. Our aim was to explore the ways in which DP might contribute to poor psychosocial functioning and occupational disability. This analysis would supply evidence relating to (a) whether there may be a need to provide support for people with DP, since no form of professional support is currently available; and (b) what specific psychosocial problems DP poses, and therefore what forms of support may be needed. We used qualitative methods to achieve our aims, since these are well suited to exploring new topics in an open manner, developing a rich, contextualized understanding of the topic and gaining insight into the diverse experiences of different individuals [14].

\section{Method}

\section{Participants}

Participants were recruited from a database associated with a website (http://www.faceblind.org) created by two of the authors $(\mathrm{BD}$ and $\mathrm{KN})$. UK residents who had registered with the website and indicated their willingness to take part in future research $(n=375)$ were invited by email to take part in a telephone interview.

To establish that these individuals had problems with face recognition, all potential participants were tested with a version of the Cambridge Face Memory Test (CFMT) [15]. This test is commonly used by many of the laboratories investigating prosopagnosia, because it has been shown to effectively discriminate between individuals with and without face memory deficits [15]. In the CFMT, subjects learn six faces at the start of the test and then must recognize those faces in novel views. There are a total of 72 items. Participants tested in the laboratory of BD $(n=9)$ were tested with the original CFMT while participants tested remotely via the internet did the CFMT II. The two versions have identical designs, similar control means and distributions (CFMT $=57.9$, S.D. $=7.9, n=50$; CFMT $\mathrm{II}=58.8$, S.D. $=9.9$, $n=36$ ), but involve different faces. Potential participants who scored 44 or below on the original CFMT were classified as prosopagnosic. In the original CFMT control group of 50 participants, only two scores were at or below this cut-off (one 44, one 43) [15].

Interviews were carried out with the first 25 individuals who met these criteria, from 106 who responded to the invitation. The scores of interviewees who had completed the original CFMT ranged from 23 to 44, with a mean of 39.0 (S.D. =9.9). The average for participants on the CFMT II was 36.5 (S.D. $=6.3$ ). These means are very similar to the mean of 37.0 (S.D. $=6.1$ ) for all DP scores on the original CFMT in published studies [3,7,16-19]. To ensure that participants with prosopagnosia acquired in adulthood were excluded, our first interview question was 'How did you come to realize you had prosopagnosia?'; none of the interviewees described adult onset (people who lose facial recognition abilities in adulthood are acutely aware of the loss) [9]. A few participants had other recognition problems (e.g., impaired recognition of objects, locations, emotions). Text from these participants was excluded wherever it was unclear whether difficulties they described were exclusively due to DP or could be related to other recognition problems; these instances were very few.

Qualitative researchers typically seek to develop an indepth understanding of a person and their context that has theoretical relevance to understanding similar people and contexts [20]. For this purpose, it is considered good practice to carry out intensive analysis of data obtained from a relatively small sample that is nonetheless sufficiently diverse to provide insight into the views and experiences of a wide range of people and contexts [21]. Confirmation that the sample is adequate is provided when analysis of the data approaches 'saturation'; i.e., the inclusion of additional data does not produce significant new insights [22]. Our participants were 18 females and 7 males aged from 26 to 74 years (mean age 48 years, S.D. 14.8). All but four were educated to at least degree level; 20 were married, 4 cohabiting, and 5 single. Analysis of data from this sample did appear to approach saturation, providing some reassurance that the sample was sufficiently large and diverse to illustrate the range of psychosocial consequences typically encountered by people with DP.

\section{Procedure}

Ethical approval was obtained from the University of Southampton School of Psychology ethics committee. Data was collected by semi-structured telephone interviews lasting between 10 and $35 \mathrm{~min}$ (mean $17 \mathrm{~min}$ ) carried out by the second and third authors. The interview schedule consisted of open-ended questions asking how the interviewee came to realize they had DP; how they felt about it; 
whether they felt it had affected their life and behavior; their perceptions of others' attitudes to them and how this might be affected by DP; whether they had had any bad experiences or emotional difficulties as a result of DP, and how they coped with these. The interviewer concluded by collecting demographic data. All interviews were tape recorded and fully transcribed.

Analysis

Thematic analysis [23,24] was used as a method of inductively identifying and understanding the psychosocial consequences of DP. The process began with immersion in the transcripts, after which initial codes describing the core meaning of each text segment were assigned to all relevant text. Related codes were grouped into themes, which were then progressively defined and refined to detail thoroughly the characteristics of each theme (e.g., variations between individuals with respect to the phenomenon described by the theme). Code definitions and the codes assigned to each text segment were fully documented [21]. This process was undertaken by the second and third authors, in discussion with the first author (who has DP). The first author then checked all final coding and prepared the overview of themes presented here, using constant comparison between cases to search for and explain significant deviations from the dominant pattern of experiences $[21,22]$.

The aim of the analysis was to document and explain the range of consequences that are commonly experienced by people with DP. The themes therefore represent experiences that were not described by many but not all participants. Qualitative research in a small nonrandom sample is unable to establish the prevalence of psychosocial consequences in people with DP, and so numerical indices of how often themes occurred could give a potentially misleading appearance of precision in estimating the impact of DP. However, qualitative descriptors are used to give a broad impression of how common the experiences were in this sample (e.g., 'most', 'many', 'some').

\section{Results}

Accounts of the psychosocial consequences of DP included numerous descriptions of the immediate consequences of recognition failure, which caused significant social interaction difficulties. Because participants felt unable to provide a legitimate justification for these difficulties, they resulted in anxiety about offending others and feelings of inadequacy. The accounts also contained descriptions of longer-lasting psychosocial consequences, including changes in behavior to avoid occurrences of recognition failure (e.g., avoidance of social situations and dependence on social support for assistance with recognition), chronic stress, and social anxiety, and a long-term impact of DP on personality, social relationships, and careers. The immediate and longer-term consequences of DP are described in more detail below.

\section{Immediate psychosocial consequences of recognition failure}

Almost all the participants told vivid stories of acutely embarrassing and often traumatic examples of recognition failure. These could take the form of greeting or joining strangers who they mistook for people they knew, or conspicuously failing to recognize familiar people-for example, introducing themselves at social gatherings to family members, longstanding friends, or work colleagues.

"I was getting off a bus and somebody got on it and grabbed me, and I pushed them out of the way, and it was only when they opened their mouth that I realized it was my own mother." (participant 02)

These events had the potential to create very difficult situations. For example, a father described his fear of trying to pick up the wrong boy when collecting his young son from school, while another participant described the sense of panic caused by knowing she would be unable to recognize her companions if she lost them in a crowd. In particular, many cases of negative reactions to violating expectations for normal social behavior were recounted. One participant was repeatedly punished as a child for failing to greet teachers outside school, and participants often reported that others had been deeply offended when they were not recognized:

"My sister said to my mother that I just looked straight through her, as if she were a stranger-but of course to me it was as if she was a stranger." (participant 24)

Consequently, a major concern of participants was that a failure of recognition would lead others to see them as unfriendly, arrogant, or discourteous:

"I think other people can often maybe find me a little bit dismissive, or think I'm not very attentive, and maybe think that they don't matter to me." (participant 14)

"If you're seen to ignore people, you can sometimes be seen as being rude, and that's never my intention, certainly I don't think I would want to behave like that, or aloof." (participant 16)

Participants therefore typically attempted to conceal their recognition problems, for example by pretending that they were preoccupied and had not noticed the other person, excusing themselves on grounds of poor eyesight or memory, or by trying to keep the conversation going until they recognized the person they were talking to. However, these strategies were frequently accompanied by anxiety that they might cause offence:

“I was just hoping she wouldn't realize I couldn't remember who she was, I just thought she'd be angry ... I just feel that I'll offend people because they'll think that I haven't paid enough attention to be bothered about who they are." (participant 18) 
Once participants knew they had DP, many found that it was useful to be able to provide an acceptable medical explanation for their difficulties. This explanation could be used to mitigate negative reactions, such as the common reaction of disbelief that people could have a facerecognition problem:

"Now I might be able to convince them a bit more, "cause if you can actually say 'Well, there's research being done in the area' then they are liable to believe one." (participant 15)

However, trying to explain the problem to everyone whom they met could itself pose a burden and was not always successful:

"I don't tell them that I've got face blindness, 'cause I think that most people won't have heard of it and, or relate to it." (participant 25)

"The children at school, I do occasionally say to them 'Look, I am terrible at faces' and they just think 'Oh, that's just an excuse."” (participant 19, school teacher)

The social interaction difficulties caused by DP therefore often provoked feelings of inadequacy, failure, and guilt, especially before the cause of their problems was known:

"I didn't talk to anyone about it 'cause I thought everybody else had this and they were managing and it was like I wasn't managing to do what everybody else was doing so I was a bit weak, and so I didn't want to let anybody know how weak I was-whereas now I don't put it down to weakness, I put it down to just the way I'm wired, and it's not a problem in terms of telling people." (participant 03)

\section{Long-term psychosocial consequences of DP}

Participants reported several common behavioral strategies for reducing the occurrence of recognition problems. These included trying to evade chance encounters and avoiding eye contact with other people:

"If I'm walking down the street and I see somebody coming towards me, and I think 'Well, I don't know if I know this person, it might be so and so, but then again it might not' then I will start fiddling in my handbag or something to avoid looking at them." (participant 07)

"Sometimes if I see someone and I'm not sure if I know them I just try and keep out of their way and hope that they don't see me, 'cause I don't know how to act." (participant 10)

It was also common to fear and avoid social gatherings:

"It [DP] makes me less interested in the social events, the partying, the getting to know lots of new people, because that just gives me a whole set of things I'll get wrong." (participant 23)

Most participants therefore expressed a preference for socialising with small groups of people they knew very well. Another commonly used coping strategy was to rely on partners or other close confidants to help participants identify people they met:

"Whenever I possibly can, I will cling to someone who knows and someone who's briefed beforehand and who will always call the person that they recognize by name to prompt me, so I know who it is that we're about to go and talk to, so it's made me quite dependent, and yet in other aspects of life I'm not at all a dependent person." (participant 04)

As the previous excerpt illustrates, these strategies for coping with DP could themselves have negative psychosocial consequences. Many participants described themselves as shy, reserved, or introverted, and one participant described herself as virtually a complete recluse. Because their face recognition problems had been lifelong, some participants were unsure to what extent their intrinsic personality or their DP had contributed to their dislike of social situations. However, many were convinced that DP had led to some degree of introversion, lack of confidence, and/or low self-esteem:

"I assumed I was stupid, I assumed I was stupid or lazy, you know all the things they labelled you with when I started school." (participant 09)

Both the direct consequences of recognition problems and the strategies used to reduce their occurrence could have lasting effects on social relationships. Clearly, avoidance of social situations could impede the formation of relationships:

"You're more comfortable in small social groups where you know everyone, or at least the one different person you know would have to be the new person, so in that sense I think it probably gives you a smaller pool of people who are friends, or, or a partner, or whatever." (participant 06)

The inability to recognize people also directly interfered with the development of relationships; participants spoke of their awareness and regret that opportunities for forming relationships were spoiled by recognition problems:

"You could get on with somebody like a house on fire, and I wasn't too bad when they were there and you were having that reinforced every day, and sort of recognize them, but of course if they went away for a couple of months and came back then it was really pretty lucky if I managed to recognize them, and they would get quite offended and it was a shame because you think 'Well, I could of, I really liked that person." (participant 17)

"I'd try, spend three days chatting up some girl and then cut her dead in the street without knowing that I'd done it." (participant 11).

In addition to harming relationships through causing offence and missing opportunities to maintain and build on social contacts, the inability to recognize an individual across a series of meetings over time could make it more difficult to create a coherent biography for them, and a sense of familiarity:

"It's hard to make a strong connection with anybody, it takes a lot of meetings ... years before you can really get to know somebody, so you become a bit excluded" (participant 08).

Concern about face recognition problems also affected choice of career and working environment, and many participants suggested that they experienced a degree of occupational disability. Certain occupations (such as policing or security) would be particularly challenging for 
people with DP. However, most occupations involve some social interactions that are made more difficult by facerecognition problems. Participants described anxiety about offending senior colleagues or valued clients by failing to acknowledge them. Greeting or networking with colleagues and customers was extremely problematic, and chairing and attending meetings with people who were known but could not be recognized were not easy. Some participants coped with these difficulties successfully, but others had chosen to avoid the stress of such situations, sometimes with a possible negative impact on their careers.

Overall, the experience of living with DP was typically described as not debilitating, but nevertheless a constant source of stress and anxiety in social situations.

"I think you spend a lot of energy actually trying to sort of just live, and so I think that's really the hardest thing, is just using a lot of energy all the time to try and remember who people are and so on. And also I think there is the fact that people do, can criticize you for the fact that you're supposed to know who they are, and you don't know who they are." (participant 21)

Naturally, the impact of DP varied depending on the personality and the circumstances of the individual, and their ability to adapt to and compensate for DP. Often DP had caused significant distress in the past, but as participants became older they found that they were more able to accept and cope with the unfortunate social consequences of recognition failure. Some participants had compensated for the social difficulties caused by DP by being friendly and outgoing to everyone (while avoiding using names), to minimize the risk of failing to greet a friend. Many participants said that DP had led them to restrict their social life, but some said this did not matter to them as they were uninterested in making a large number of social contacts. However, others felt that the inability to establish a wide social network had resulted in lack of confidence and isolation.

\section{Discussion}

This study sought to describe the ways in which DP might contribute to poor psychosocial functioning and occupational disability, in order to identify what forms of support may be needed by people with DP. Our participants all recounted recurring social interaction difficulties, some evidently traumatic. The inability to offer a socially acceptable explanation for recognition failure led to anxiety that others would perceive them as disrespectful or uncaring, often resulting in some degree of fear and avoidance of situations involving meeting people. Long-term consequences could include loss of self-confidence, dependence on others, a restricted social circle, and more limited employment opportunities.

Social anxiety disorder is characterized by a marked and persistent fear that certain social situations will cause embarrassment, which results in fear and avoidance of these situations and interferes with normal activities [11]. From the accounts of people with DP, it seems clear that DP must pose a risk factor for the development of some of the central features of social anxiety disorder. This diagnosis can only be made in the absence of medical conditions that can interfere with social functioning, and people with DP are only likely to be predisposed to some aspects of social anxiety disorder (i.e., anxiety about social interaction rather than performance) [25]. Nevertheless, the general mental and physical health of people who exhibit some but not all features of social anxiety disorder is significantly worse than the general population, and not much better than people with diagnosed social anxiety disorder [26]. Recent research suggests that mental and physical health can be negatively affected when individuals feel that they are obliged to exert high levels of effort in social relationships but these do not reliably lead to social reward and a sense of belonging [27].

Cognitive behavioral therapy and social skills training are known to be helpful in social anxiety disorder [10] and might assist people to cope with the difficulties posed by DP. Whereas our analysis identified some strategies for coping with DP (such as restricting social contact) that might exacerbate psychological difficulties, other coping strategies (e.g., selective disclosure) may be adaptive. Our findings also suggest that the personality and social circumstances of the individual moderate the impact of DP; consequently, while the majority are unlikely to require clinical intervention, there may be particular subgroups of people with DP who could benefit from psychological support. In particular, support may be useful for young people who need to establish a new set of social relationships at school, college, university, or work, if they lack confidence in managing the social difficulties caused by DP. Prosopagnosia may also contribute to the difficulties of some people with autism spectrum disorders [12].

The psychosocial consequences of DP have parallels with those experienced by people with other conditions that can be partially concealed, but if revealed may result in social embarrassment or humiliation. People with conditions that affect their appearance, such as psoriasis or excess hair, report low self-confidence and avoid social situations because of anticipation of negative social reactions [28,29]. People who stutter [30,31] or have dyslexia [32-34] also often fear and avoid social situations which may expose their difficulties and report anxiety and low self-esteem, and damage to career prospects. As in DP, people with dyslexia report that confirmation that their difficulties are due to a neurological condition can help to alleviate the sense of personal failure [33]. Similarly, people who stutter can reduce the strain of social encounters by disclosing their difficulties in advance [31]. However, like those with DP, people with dyslexia felt that disclosure could have negative consequences and were concerned that a diagnosis could evoke stigmatising stereotypes of mental deficiency [33,34]. 
Despite the problem of stigmatization, many people with dyslexia welcome the access to support that can be gained by explicit recognition of their problems $[33,34]$. People with DP describe widespread occupational difficulties, but have previously had no such access to recognition and support. If the social interaction difficulties caused by DP are formally acknowledged as a consequence of an impairment, then they have a right to expect employers and educators to make 'reasonable adjustments' to allow for them. For example, the US National Stuttering Association Employer's Handbook [35] advises employers that in some jobs it may be reasonable to exempt people who stutter from taking telephone calls and provides information about how to help people who stutter fulfil their potential. Equivalent support might be useful to people with DP.

This study was carried out in a small, unrepresentative sample of people with DP; it is not possible to tell how typical their experiences are of the wider population. The nature of the difficulties described in their accounts, and the range of responses to DP, seems similar in nature to published case histories of people with DP, which include reports of welladapted and highly successful people as well as some who appear distressed and handicapped by DP $[6,9,36,37]$. Nevertheless, it is possible that those who registered with the website and volunteered to be interviewed tended to be more concerned about their face recognition problems than those who did not. However, this sample overrepresented highly educated people, due to recruitment via a universitybased website. It is therefore also possible that this highly educated sample overrepresented people who were particularly well placed to cope with DP. Although common themes emerged across participants with very diverse histories and characteristics, quantitative research in a representative sample and matched control group is needed to establish the prevalence among people with DP of the phenomena identified in this study, and whether occupational difficulties and symptoms of social anxiety disorder are more common than in the general population.

\section{Conclusions}

Prosopagnosia clearly causes considerable difficulties for social interaction, which can have a lasting negative impact on social relations and psychological well-being. Many people with DP learn to adapt to and overcome these difficulties to a remarkable extent; indeed, a former UK Prime Minister is believed to have had DP [36]. Nevertheless, the potential for negative psychosocial consequences and occupational disability posed by DP is as great as that posed by conditions which are currently afforded explicit professional recognition and support, such as stuttering and dyslexia. As the title of this paper implies, recognition of the problems posed by DP could help to reduce its impact. Greater public awareness of the condition would make it easier to explain and justify social interaction difficulties to other people, including employers. Greater professional awareness could facilitate appropriate detection and referral of those who may require social skills training and/or treatment for social anxiety disorder.

\section{Acknowledgments}

We would like to thank the participants and Laura Germine for help with recruitment.

\section{References}

[1] Duchaine BC, Nakayama K. Developmental prosopagnosia: a window to content-specific face processing. Curr Opin Neurobiol 2006;16: $166-73$.

[2] Behrmann M, Thomas C, Humphreys K. Seeing it differently: visual processing in autism. Trends Cogn Sci 2006;10:258-64.

[3] Duchaine B, Germine L, Nakayama K. Family resemblance: ten family members with prosopagnosia and within-class object agnosia. Cogn Neuropsychol 2007;24:419-30.

[4] Kennerknecht I, Grueter T, Welling B, Wentzek S, Horst J, Edwards S, Grueter M. First report of prevalence of non-syndromic hereditary prosopagnosia (HPA). Am J Med Genet 2006;140:1617-22.

[5] Le Grand R, Mondloch CJ, Maurer D, Brent HP. Expert face processing requires visual input to the right hemisphere during infancy. Nat Neurosci 2003;6:1108-12.

[6] Duchaine BC. Developmental prosopagnosia with normal configural processing. NeuroReport 2000;11:79-83.

[7] Duchaine B, Nakayama K. Dissociations of face and object recognition in developmental prosopagnosia. J Cogn Neurosci 2005;17:249-61.

[8] Barton J. Disorders of face perception and recognition. Neurol Clin 2003;21:521-48.

[9] Bornstein B. Prosopagnosia. In: Halpern L, editor. Problems of dynamic neurology. Jerusalem: Hadassah Medical School, 1963. pp. 283-318.

[10] Antony MM. Assessment and treatment of social phobia. Can J Psych 1997;42:826-34.

[11] American Psychiatric Association. Diagnostic and statistical manual of mental disorders: DSM-IV-TR. Washington, DC: American Psychiatric Association, 2000.

[12] Barton JJ, Cherkasova MV, Hefter R, Cox TA, O'Connor M, Manoach DS. Are patients with social developmental disorders prosopagnosic? Perceptual heterogeneity in the Asperger and socio-emotional processing disorders. Brain 2004;127:8-16.

[13] Schultz RT. Developmental deficits in social perception in autism: the role of the amygdala and fusiform face area. Int J Dev Neurosci 2005;23:125-41

[14] Camic PM, Rhodes JE, Yardley L. Naming the stars: integrating qualitative methods into psychological research. In: Camic PM, Rhodes JE, Yardley L, editors. Qualitative research in psychology: expanding perspectives in methodology and design. Washington, DC: American Psychological Association, 2003. pp. 3-15.

[15] Duchaine B, Nakayama K. The Cambridge Face Memory Test: results for neurologically intact individuals and an investigation of its validity using inverted face stimuli and prosopagnosic participants. Neuropsychologia 2006;44:576-85.

[16] Bentin S, DeGutis JM, D'Esposito M, Robertson LC. Too many trees to see the forest: performance, ERP and fMRI manifestations of integrative congenital prosopagnosia. J Cogn Neurosci 2007;19:132-46.

[17] Duchaine BC, Yovel G, Butterworth E, Nakayama K. Prosopagnosia as an impairment to face-specific mechanisms: elimination of the alternative hypotheses in a developmental case. Cogn Neuropsychol 2006;23:714-47.

[18] Garrido L, Duchaine BC, Nakayama K. Face detection in normal and prosopagnosic individuals. J Neuropsychol 2008. 
[19] Duchaine B, Yovel G, Nakayama K. No global processing deficit in the Navon task in 14 developmental prosopagnosics. Soc Cogn Affect Neurosci 2007;2:104-13.

[20] Johnson JM. Generalizability in qualitative research: excavating the discourse. In: Morse JM, editor. Completing a qualitative project: details and dialogue. London: Sage, 1997. pp. 191-208.

[21] Yardley L. Demonstrating validity in qualitative psychology. In: Smith JA, editor. Qualitative Psychology. 2nd ed. London: Sage, 2008.

[22] Chamberlain K, Camic P, Yardley L. Qualitative analysis of experience: grounded theory and case studies. In: Marks DF, Yardley L, editors. Research methods for clinical and health psychology. London: Sage, 2004. pp. 69-89.

[23] Joffe H, Yardley L. Content and thematic analysis. In: Marks D, Yardley L, editors. Research methods for clinical and health psychology. London: Sage, 2004. pp. 56-68.

[24] Braun V, Clarke V. Using thematic analysis in psychology. Qual Res Psychol 2006;3:77-101.

[25] Safren SA, Turk CL, Heimberg RG. Factor structure of the Social Interaction Anxiety Scale and the Social Phobia Scale. Behav Res Ther 1998;36:443-53.

[26] Davidson JR, Hughes DC, George LK, Blazer DG. The boundary of social phobia. Exploring the threshold. Arch Gen Psychiatr 1994;51:975-83.

[27] Chandola T, Marmot M, Siegrist J. Failed reciprocity in close social relationships and health: findings from the Whitehall II study. J Psychosom Res 2007;63:403-11.
[28] Fox FE, Rumsey N, Morris M. "Ur skin is the thing that everyone sees and you cant change it!": exploring the appearance-related concerns of young people with psoriasis. Dev Neurorehabil 2007;10: $133-41$.

[29] Lipton MG, Sherr L, Elford J, Rustin MH, Clayton WJ. Women living with facial hair: the psychological and behavioral burden. J Psychosom Res 2006;61:161-8.

[30] Corcoran JA, Stewart M. Stories of stuttering: a qualitative analysis of interview narratives. J Fluency Disord 1998;23:247-64.

[31] Crichton-Smith I. Communicating in the real world: accounts from people who stammer. J Fluency Disord 2002;27:333-51.

[32] Alexander-Passe N. How dyslexic teenagers cope: an investigation of self-esteem, coping and depression. Dyslexia 2006;12: 256-75.

[33] Dale M, Taylor B. How adult learners make sense of their dyslexia. Disabil Soc 2001;16:997-1008.

[34] Morris D, Turnbull P. Clinical experiences of students with dyslexia. J Adv Nurs 2006;54:238-47.

[35] National Stuttering Association. What employers should know about stuttering. Available from http://www.nsastutter.org/pdfs/newsletters/ s_212.pdf; [cited 2007 Nov 31].

[36] Gruter T, Gruter M. Prosopagnosia in biographies and autobiographies. Perception 2007;36:299-301.

[37] Dobel C, Bölte J, Aicher M, Schweinberger SR. Prosopagnosia without apparent cause: overview and diagnosis of six cases. Cortex 2007;43:718-33. 